The median patient age was 52 years, and the median time from organ transplantation to cardiac surgery was 79 months. For kidney recipients, the 1-year and 5-year patient survival rates after cardiac surgery were $97 \%$ and $82 \%$, respectively. After a mean followup of 39 months, median New York Heart Association functional class improved from class 3 to class 1 across the cohort. Non-graftrelated complications occurred in 17 patients; respiratory and cardiovascular problems were the most common. Allograft dysfunction requiring dialysis occurred in two kidney recipients, one of whom required dialysis following discharge. Three other patients had transient renal graft dysfunction soon after cardiac surgery. During follow-up, three patients suffered renal graft failure, two of whom had required emergency cardiac surgery. One patient died within 30 days of surgery. At last follow-up, 12 kidney transplantees had died, two as a result of cardiac events.

The authors conclude that cardiac surgery can be safely performed in kidney graft recipients, although comorbid conditions and immunosuppression place the patients at greater risk of perioperative complications. There might be a link between emergency cardiac surgery and subsequent graft failure.

Original article Deb SJ et al. (2006) Cardiac surgery in kidney and liver transplant recipients. Mayo Clin Proc 81: 917-922

\section{High prevalence of glomerulopathy in sickle cell anemia}

A recent study has shown that the prevalence of glomerular damage in adults with various forms of sickle cell anemia is much higher than previously thought.

Guasch and colleagues investigated the prevalence of glomerular damage and clinical correlates of renal failure in 300 adults with the homozygously inherited, clinically severe hemoglobin SS disease $(n=184)$ and other sickling hemoglobinopathies (a total of 116 patients with the heterozygously inherited, generally lesssevere SC or SD disease, or S $\beta$ thalassemia of variable severity). The albumin excretion rate of patients with SS disease was more likely to be increased than that of patients with SC disease, $\mathrm{SD}$ disease, or $\mathrm{S} \beta$ thalassemia (68\% vs $32 \%$ ).
Albuminuria tended to be associated with age and serum creatinine level in SS disease, and with age in other sickling disorders. There was no association between albuminuria and blood pressure in any patient group, and significant hypertension did not affect the $21 \%$ of patients with SS disease and renal insufficiency. Given this lack of association, the authors question the use of antihypertensive therapy per se in sufferers of sickle cell anemia, and suggest that targeting proteinuria, for example, might be more effective.

No differences in hematological parameters—including hemoglobin level—between patients with normal and abnormal albumin levels were detected. This finding implies that the mechanisms underlying development of glomerular damage in sickling hemoglobinopathies might not be solely related to chronic anemia.

Original article Guasch A et al. (2006) Glomerular involvement in adults with sickle cell hemoglobinopathies: prevalence and clinical correlates of progressive renal failure. J Am Soc Nephrol 17: 2228-2235

\section{ARF-specific scoring methods do not predict in-hospital mortality}

Critically ill patients with acute renal failure (ARF) generally have a poor outcome. There is no standardized definition of ARF, however, which has produced variance in reported mortality rates and made comparison between studies difficult. Åhlström and colleagues evaluated the ability of two recently proposed ARF-specific scoring systems, the Acute Dialysis Quality Initiative Risk, Injury, Failure, Loss, End-Stage Renal Disease (RIFLE) consensus classification and Bellomo et al.'s 2001 classification, to predict in-hospital mortality in critically ill patients, and compared them with APACHE II and SOFA, two general severity-of-illness scoring systems.

ARF prevalence was determined in 658 consecutive patients treated in two intensive care units. Increasing stage of ARF according to the ARF-specific scoring methods correlated with increasing mortality. Admission SOFA scores and maximum RIFLE scores for the first 3 days in intensive care independently predicted in-hospital mortality in forward conditional logistic regression analyses; in receiver 